\title{
A Pilot Study of Novel Biomarkers in Neonates With Hypoxic-Ischemic Encephalopathy
}

\author{
MARTHA DOUGLAS-ESCOBAR, CUI YANG, JEFFREY BENNETT, JONATHAN SHUSTER, DOUGLAS THERIAQUE, \\ AVITAL LEIBOVICI, DAVID KAYS, TONG ZHENG, CANDACE ROSSIGNOL, GERRY SHAW, AND MICHAEL D. WEISS
}

\begin{abstract}
Department of Pediatrics [M.D.-E.], Baylor College of Medicine, Houston, Texas 77030; Departments of Neuroscience [C.Y., T.Z., G.S.], Neuroradiology [J.B.], Epidemiology and Health Policy Research [J.S.], Pediatrics [A.L., C.R., M.D.W.], and Pediatric Surgery [D.K.], University of Florida, Gainesville, Florida 32611; Clinical and Translational Science Institute [J.S., D.T.], University of Florida, Gainesville, Florida 32610
\end{abstract}

\begin{abstract}
Severe hypoxic-ischemic encephalopathy (HIE) is a devastating condition that can lead to mortality and long-term disabilities in term newborns. No rapid and reliable laboratory test exists to assess the degree of neuronal injury in these patients. We propose two possible biomarkers: 1) phosphorylated axonal neurofilament heavy chain (pNF-H) protein, one of the major subunits of neurofilaments, found only in axonal cytoskeleton of neurons and 2) Ubiquitin C-terminal hydrolase 1 (UCHL1 protein) that is heavily and specifically concentrated in neuronal perikarya and dendrites. High-serum pNF-H and UCHL1 levels are reported in subarachnoid hemorrhage and traumatic brain injury, suggesting that they are released into blood following neuronal injury. We hypothesized that serum pNF-H and UCHL1 were higher in neonates with moderateto-severe HIE than in healthy neonates. A time-limited enrollment of 14 consecutive patients with HIE and 14 healthy controls was performed. UCHL1 and pNF-H were correlated with clinical data and brain MRI. UCHL1 and pNF-H serum levels were higher in HIE versus controls. UCHL1 showed correlation with the 10-min Apgar score, and pNF-H showed correlation with abnormal brain MRI. Our findings suggest that serum UCHL1 and pNF-H could be explored as diagnostic and prognostic tools in neonatal HIE. (Pediatr Res 68: 531-536, 2010)
\end{abstract}

A lthough several biomarkers have shown some promise $(1,2)$, no rapid and reliable serum marker is currently available to detect the extent of acute neurologic injury in the neonate. Because no readily available, sensitive, and specific test exists, the degree of neuronal injury is typically assessed by the physical examination (3) and by the neuroimaging studies. The development of a rapid test would enable the clinician to accurately gauge the initial degree of injury and objectively follow the progression overtime. This information would guide treatment decisions and therapies, such as hypothermia, in an objective and selective manner and also de-

Received January 22, 2010; accepted July 27, 2010

Correspondence: Martha Douglas-Escobar, M.D., Department of Pediatrics, Baylor College of Medicine, 6621 Fannin MC WT 6-145, Houston, TX 77030; e-mail: douglase@bcm.edu

Supported, in part, by Grants M01RR00082 and U54RR025208 from the National Institute of Research Resources and National Institutes of Health and by Grant NS02198 [M.D.W.]. crease complications in patients who would not benefit from the treatment $(4,5)$.

Two potential biomarkers of neurological injury are the phosphorylated axonal neurofilament heavy chain ( $\mathrm{pNF}-\mathrm{H}$ ) and ubiquitin C-terminal hydrolase 1 (UCHL1). The NF-H protein is one of the major subunits of neurofilaments, the main components of the axonal cytoskeleton (6). Because neurofilaments are found only in neurons, antibodies to them are widely used to identify neurons and their processes. We focused on a phosphorylated form of NF-H (pNF-H) that is specific for axons. Importantly, $\mathrm{pNF}-\mathrm{H}$ found in patient fluids can only originate from the axons of neurons, indicating ongoing axonal damage or degeneration. Our previous series of reports showed that the serum and cerebrospinal fluid (CSF) levels of pNF-H are increased following a variety of CNS damage and disease states, both in animal models and in human patients (7-11).

More recently, we showed that the neuron-specific cytoplasmic enzyme ubiquitin $\mathrm{C}$ terminal hydrolase 1 (UCHL1) is a proteolytically stable and abundant protein, readily detected in the CSF of patients recovering from aneurysmal subarachnoid hemorrhage (12). Higher levels of UCHL1 in CSF had been reported after traumatic brain injury and surgically induced circulatory arrest $(13,14)$. Measurement of UCHL1 levels provides a measure of neuronal injury potentially complementary to that of pNF-H, because UCHL1 is heavily concentrated in neuronal perikarya and dendrites $(15,16)$.

This pilot project examined the serum levels of $\mathrm{pNF}-\mathrm{H}$ and UCHL1 in patients with neonatal hypoxic-ischemic encephalopathy (HIE) and normal controls. We hypothesized that the serum levels of $\mathrm{pNF}-\mathrm{H}$ and UCHL1 from neonates with moderate-to-severe HIE would be detectable and higher when compared with control newborns.

\section{MATERIALS AND METHODS}

Patient population and sampling protocol. All aspects of this study were approved by the University of Florida Institutional Review Board, and

Abbreviations: CSF, cerebrospinal fluid; CV, coefficient of variance; HIE, hypoxic ischemic encephalopathy; HRP, horse radish peroxidase; pNF-H, phosphorylated axonal neurofilament heavy chain; ROC, receiver operating characteristic; UCHL1, ubiquitin C-terminal hydrolase 1 
patients were enrolled after obtaining the informed consent from the parents, at Shands University of Florida Teaching hospital (January 2006-January 2009). We enrolled 14 consecutive cases of neonates with HIE, with GA $\geq 38$ wk admitted to the intensive care unit between birth and $72 \mathrm{~h}$ of age. Inclusion criteria for a patient with HIE included metabolic acidosis, Apgar $<3$ at $5 \mathrm{~min}$ of age, neurological symptoms (e.g. seizures, coma, and hypotonia), multiple organ involvement (e.g. kidney, liver, heart, intestine, and lungs), and physical examination compatible with moderate-to-severe HIE by Sarnat scores II or III on admission. Exclusion criteria for neonates with HIE were transferred after $72 \mathrm{~h}$ of birth, physical examination compatible with mild HIE (by Sarnat scores I) on admission, and inborn errors of metabolism or congenital anomalies. In 2009, our neonatal intensive care unit started a hypothermia protocol for moderate-to-severe patients with HIE, and as a result, the last five patients with HIE who enrolled received hypothermia treatment. We enrolled 14 control neonates with GA $\geq 38$ wk who were admitted to the Well-Baby Newborn Nursery. Neonates in the control group were excluded if they were diagnosed with inborn errors of metabolism, congenital malformations, hypoglycemia, or undergoing an evaluation for risk factors for sepsis.

Blood samples. We obtained $2 \mathrm{~mL}$ of blood from the neonates with HIE at multiple time points during the first $3 \mathrm{~d}$. Sarnat scoring was performed at 0-6, 7-12, 13-24, 25-48, and 49-72 h after birth, and blood samples were obtained immediately after scoring, as often as possible. The first sample was obtained at the time of admission to the newborn intensive care unit. In the control neonates, a single sample of blood $(500 \mu \mathrm{L})$ was collected at the time of the sample for the neonatal metabolic screen between 24 and $48 \mathrm{~h}$ of age.

ELISA. Blood samples were assayed for the presence of $\mathrm{pNF}-\mathrm{H}$ and UCHL1 using appropriate ELISAs. A two MAb sandwich using MAb specific for pNF-H was recently described (8). Briefly, the AH1 pNF-H capture antibody was used along with the NAP4 detection antibody that was directly labeled with horse radish peroxidase (HRP). NAP4 was originally raised against a preparation of pig pNF-H as described (17). Both, AH1 and NAP4 specifically recognize only the phosphorylated axonal form of NF-H protein, and both antibodies bind only to axonal profiles on neurons in tissue culture and in brain sections. This assay detects pNF-H with the lowest cutoff of 30 $\mathrm{pg} / \mathrm{mL}$ and an average interassay coefficient of variance $(\mathrm{CV})$ of 0.057 . The intraassay $\mathrm{CV}$ is between 0.125 and 0.085 . For UCHL1 ELISA, we used a single monoclonal capture antibody for UCHL1 and clone $\mathrm{BH} 7$ for capture and detection an affinity purified rabbit polyclonal as described (12). Both antibodies were raised against recombinant human UCHL1 expressed in and purified from E. coli. This assay can detect down to $50 \mathrm{pg} / \mathrm{mL} \mathrm{UCHL1}$ and has an interassay $\mathrm{CV}$ of 0.053 .

$\mathrm{MAb}$ AH1 and BH7 were affinity purified on protein A/G sepharose (Sigma Chemical Co.-Aldrich, St. Louis, MO) and then applied overnight to Immulon $4 \mathrm{HBX}$ plates at $1 \mu \mathrm{g} / \mathrm{mL}$ in $50 \mu \mathrm{L}$ of $50-\mathrm{mM}$ sodium bicarbonate buffer at $\mathrm{pH}=9.5$. The plates were blocked in $2 \%$ nonfat milk in Trisbuffered saline (TBS) for $1 \mathrm{~h}$ at room temperature and then $20 \mu \mathrm{L}$ of plasma was added with the volume made up to $50 \mu \mathrm{L}$ with $2 \%$ nonfat milk in TBS plus $0.1 \%$ Tween 20 . Serial dilutions of appropriate protein standards were also applied to a row on each plate, typically with final concentrations per well from $5 \mathrm{ng} / \mathrm{mL}$ to $10 \mathrm{pg} / \mathrm{mL}$ and with a blank that contained no standard but was incubated with the various antibody reagents. Standards were either bovine pNF-H purified from bovine spinal cord (11) or recombinant HIStagged human UCHL1 (12). After incubation on a shaker for $1 \mathrm{~h}$ at room temperature, pNF-H plates were washed on a BioTek plate washer and 100 $\mu \mathrm{L}$ of $1 \mu \mathrm{g} / \mathrm{mL}$ NAP4-HRP conjugate detection antibody was added. After incubation for $1 \mathrm{~h}$ at room temperature with gentle shaking, the plate was again washed and 3,3',5,5' -tetramethylbenzidine (TMB) peroxidase substrate was added and the development was allowed to proceed for $20 \mathrm{~min}$ at room temperature. This version of the UCHL1 assay used an affinity purified rabbit antibody followed by commercial goat anti-rabbit HRP (Sigma Chemical Co-Aldrich), which required an extra cycle of incubation and washing, followed by TMB peroxidase for $20 \mathrm{~min}$ at room temperature. The HRP reaction product was measured with a BioTek ELISA plate reader at 450-nm absorbance, and data were collected in an Excel spreadsheet. All the UCHL1 and pNF-H antibodies used in this study are commercially available from EnCor Biotechnology Inc. (Gainesville, FL).

Severity of injury and outcome measurements. All the neonates with HIE had a neurologic examination performed with a Sarnat score assigned before blood sampling. The same observer performed all the Sarnat scorings and the neonates were categorized based on the highest level of any of the Sarnat categories tested. For example, if tone was judged to be in the category III, the neonate was assigned a Sarnat score of III even if other categories were compatible with a score of II. Other data collected included patient demographic information and neuroimaging results. The neuroimaging studies consisted of an MRI performed according to clinical indications between $24 \mathrm{~h}$ and $5 \mathrm{~d}$ of age. The studies were performed on a Magnetom Sonata 1.5T scanner (Siemens Medical Solutions, Erlangen, Germany). The protocol included axial T1, axial T2, and diffusion-weighted sequences including $b=$ $0, b=1000$, and apparent diffusion coefficient map. All the MRIs were read by a single subspecialty board-certified neuroradiologist (J.B.) with 7 years of experience interpreting neonatal brain MRIs, who was blinded to the clinical severity of HIE. Findings were assigned to four major categories (normal, or injury classified as diffuse cortical, focal cortical, basal ganglia/hippocampus/ thalamus). This information was entered into a centralized database for analysis to determine correlations between the various clinical variables and the level of serum biomarkers.

Statistical methods. This work was designed to serve as a time-limited exploratory study during the 36-mo period from January 2006 through January 2009. We enrolled 14 consecutive patients with HIE and 14 healthy controls. Because of the small sample size and a propensity for outliers in some of the variables, all inferential analyses used two-sided nonparametric rank-based tests. Descriptive statistics were reported as medians, quartiles, and range. Cases and controls were compared with the Wilcoxon test. Because controls contribute a single observation, we only used the first available marker for the cases with HIE to compare with the controls.

To test for markers as increasing or decreasing with time, for each subject who had at least two serial marker values, we computed a slope by least squares and subjected the slopes to a Wilcoxon sign-rank test to test the null hypothesis of a stable biomarker overtime. Finally, we assessed the association between the earliest marker and baseline values via the Spearman correlation coefficient.

\section{RESULTS}

Demographic information. The demographic information for neonates with HIE and control neonates is shown in Table 1. GA, ethnicity, and birth weight were comparable for the two groups. Female neonates were predominant in the HIE group, and $\sim 70 \%$ were outborn and referred for treatment to the Shands Teaching Hospital. Clinical variables in patients with HIE are shown in Table 2.

Serum levels of pNF-H and UCHL1. We obtained a total number of 43 samples from patients with HIE, and not all time points were available on every enrolled patient with HIE (0-6 h, $n=5 ; 7-12 \mathrm{~h}, n=7 ; 13-24 \mathrm{~h}, n=12 ; 25-48 \mathrm{~h}, n=11$; and $49-72 \mathrm{~h}, n=8$ ). Average serum levels in the first available sample from each patient of both UCHL1 and pNF-H were higher in patients with HIE when compared with the controls (Fig. 1). The median of the first serum level of UCHL1 in neonates with HIE was $2.8 \mathrm{ng} / \mathrm{mL}$ (quartiles, $Q 1=$ 1.6 and $Q 3=22.7$; range, $170.4-0.4 \mathrm{ng} / \mathrm{mL}$ ) compared with

Table 1. Demographic and clinical information from HIE and control patients

\begin{tabular}{lcc}
\multicolumn{1}{c}{ Data } & $\begin{array}{c}\text { HIE } \\
(n=14)\end{array}$ & $\begin{array}{c}\text { Control } \\
(n=14)\end{array}$ \\
\hline GAs (wk) & $38.6( \pm 1.6)$ & $38.9( \pm 1.3)$ \\
Birth weights $(\mathrm{g})$ & $3125( \pm 450)$ & $3425( \pm 604)$ \\
Gender (\%) & & \\
$\quad$ Female & 64 & 36 \\
Ethnicity (\%) & & \\
$\quad$ Caucasian, non-Hispanic & 50 & 46 \\
$\quad$ Hispanic & 21 & 23 \\
$\quad$ African-American & 29 & 24 \\
$\quad$ Asian & 0 & 7 \\
Outborn (\%) & 71 & 0 \\
Apgar score & & $7.6( \pm 1.9)$ \\
1 min & $1.4( \pm 1.9)$ & $8.5( \pm 1.1)$ \\
5 min & $2.7( \pm 2.8)$ & NA \\
10 min & $3.7( \pm 3.1)$ & \\
\hline
\end{tabular}

There were $N=14$ subjects in each groups. Variables presented as means $\pm \mathrm{SD}$, gender, ethnicity, and outborn are given in percentages.

NA, not assayed. 
Table 2. Clinical information from HIE and control patients

\begin{tabular}{lc}
\multicolumn{1}{c}{$\begin{array}{c}\text { Clinical data in } \\
\text { HIE patients }\end{array}$} & $n=14(100 \%)$ \\
\hline Admitted for & $10(71)$ \\
Labor & $2(14.2)$ \\
Rupture of membranes & $5(35.7)$ \\
Pregnancy complications & $0(0)$ \\
Delivered at home & $1(7.1)$ \\
Fever $\left(>38^{\circ} \mathrm{C}\right)$ during labor or chorioamnionitis & $7(50)$ \\
Induction or augmentation of labor with Pitocin & $6(42.9)$ \\
Meconium stained amniotic fluid & \\
Mode of delivery & $4(28.6)$ \\
Spontaneous vaginal & $1(7.1)$ \\
Instrumental delivery & $9(64.3)$ \\
Cesarean section & \\
Indication for operative delivery & $2(14.2)$ \\
Abruptio placenta & $1(7.1)$ \\
Cord prolapsed/vasa previa & $11(78.6)$ \\
Fetal distress & $1(7.1)$ \\
Dystocia & $10.9( \pm 13.5)$ \\
Base deficit (average) & $64 \%$ \\
Base deficit $>12$ & $7.15( \pm 0.15)$ \\
First pH (average) & $50 \%$ \\
pH $<7.1$ & $10.3( \pm 4.9)$ \\
Blood lactate & $35.7 \%$ \\
Hypothermia &
\end{tabular}

Variables are expressed \pm SD for base deficit, first $\mathrm{pH}$, and blood lactate. The other variables are presented in percentages.
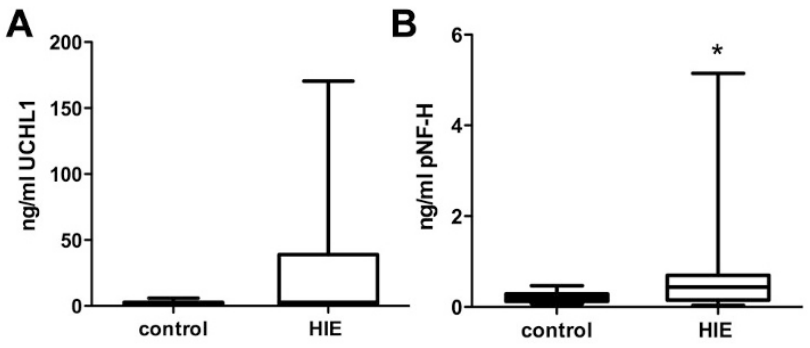

Figure 1. Serum biomarkers collected from the first blood sample of patients with HIE $v s$ control subjects. Panel $(A)$ shows UCHL-1, and panel $(B)$ shows pNF-H. Box and whiskers plots indicate quartiles and range of data and * indicates $p<0.05$

the control median of $1.7 \mathrm{ng} / \mathrm{mL}(Q 1=1.2$ and $Q 3=2.7$; range, $5.9-0.69 \mathrm{ng} / \mathrm{mL} ; p=0.10$, by two-sided Wilcoxon test). The median of the first serum pNF-H level was 0.44 $\mathrm{ng} / \mathrm{mL}(Q 1=0.17$ and $Q 3=0.60$; range, $5.14-0.03 \mathrm{ng} / \mathrm{mL})$ in HIE patients compared with $0.015 \mathrm{ng} / \mathrm{mL}(Q 1=0.13$ and $Q 3=0.28$; range, $0.46-0.07 \mathrm{ng} / \mathrm{mL})$ in controls $(p=0.051$ by two-sided Wilcoxon test). UCHL1 levels in some patients were surprisingly high, with peaks of $170.4 \mathrm{ng} / \mathrm{mL}$ in one patient and $155.2 \mathrm{ng} / \mathrm{mL}$ in another patient, both of whom subsequently died (Fig. 2, panels $A$ and $B$ ). The peak levels of pNF-H were 5.15 and $2.43 \mathrm{ng} / \mathrm{mL}$ in two patients, one of whom died (Fig. 2C). We constructed receiver operating characteristic (ROC) curves of all data for both biomarkers with the patients dichotomized between the three who died and the remainder (Fig. 2D). The area under the curve for UCHL1 was 0.931 and for pNF-H was 0.755 , and $100 \%$ specificity was achieved at $\sim 131 \mathrm{ng} / \mathrm{mL}$ for UCHL1 and $\sim 0.71 \mathrm{ng} / \mathrm{mL}$ for pNF-H. Ninety-five percent specificity was achieved at $\sim 28 \mathrm{ng} / \mathrm{mL}$ for UCHL1 and $\sim 0.28 \mathrm{ng} / \mathrm{mL}$ for pNF-H.

Correlation between serum biomarkers and clinical findings. Table 3 summarizes the HIE patients' Apgar scores at 1,5 , and $10 \mathrm{~min}$, initial arterial $\mathrm{pH}$, and MRI pattern of injury. The highest level of UCHL1 and pNF-H are also shown with the time point collected. A Spearman correlation coefficient analysis was performed using the earliest biomarker to examine the relationships between the biomarker levels and the Apgar scores at $10 \mathrm{~min}$, blood lactate levels, and the initial arterial $\mathrm{pH}$. There was a strong negative correlation between serum level of UCHL1 and Apgar score at $10 \mathrm{~min}$ $(p=0.003$, Fig. $2 E)$ and the initial neonatal arterial $\mathrm{pH}(p=$ 0.003 , Fig. $2 F$ ). However, there was no correlation between initial blood pNF-H level and either Apgar score at 10 min or initial arterial $\mathrm{pH}$. There was also no significant relationship between improving or worsening Sarnat scores and the changes in the levels of either pNF-H or UCHL1 overtime.

There is a correlation between abnormal MRI and higher initial serum pNF-H, because the three patients with the highest pNF-H level all had abnormal MRIs (Fig. 3A). When we categorized the brain MRI into normal and three pathologic groups (Fig. $3 B$ ), we found that pNF-H serum concentrations tended to be highest in patients who died and had a basal ganglia/hippocampus/thalamus injury whereas patients who died or had diffused cortical injuries had higher serum UCHL1 concentrations. However, three patients with HIE with apparently normal initial scans also had high levels of UCHL1.

\section{DISCUSSION}

In this pilot study, we showed that serum UCHL1 and pNF-H are detectable in the serum of patients with HIE. Although UCHL1 levels have been studied in a limited extent in CSF, this is the first report of UCHL1 detection in blood after the CNS injury as far as we are aware. The most striking finding was that the highest levels of UCHL1 were detected in the serum of patients who subsequently died. The high level of UCHL1 detected, $>100 \mathrm{ng} / \mathrm{mL}$ in some cases, suggests that detection of this protein might form the basis of a rapid screening assay. We also noted that the increased levels of serum pNF-H were predictive of poor outcome, although levels of serum pNF-H were much lower than the levels of UCHL1 and pNF-H determination that seemed to be significantly less specific. However, both biomarkers show promise, and we intend to perform more extensive studies with much larger numbers of patients in future.

UCHL1, originally known as Pgp9.5, is an abundant protein localized exclusively to neurons and neuroendocrine cells and concentrated in perikarya and dendrites (15). We recently showed that UCHL1 is resistant to endogenous brain and serum proteases, a highly desirable trait for a potential biomarker (12). Because UCHL1 is concentrated in the cortex, we propose that sudden release of this protein into blood represents primarily acute cortical damage in these patients. Therefore, UCHL1 measurement seems to show promise in the detection of acute cortical damage in neonatal patients with 

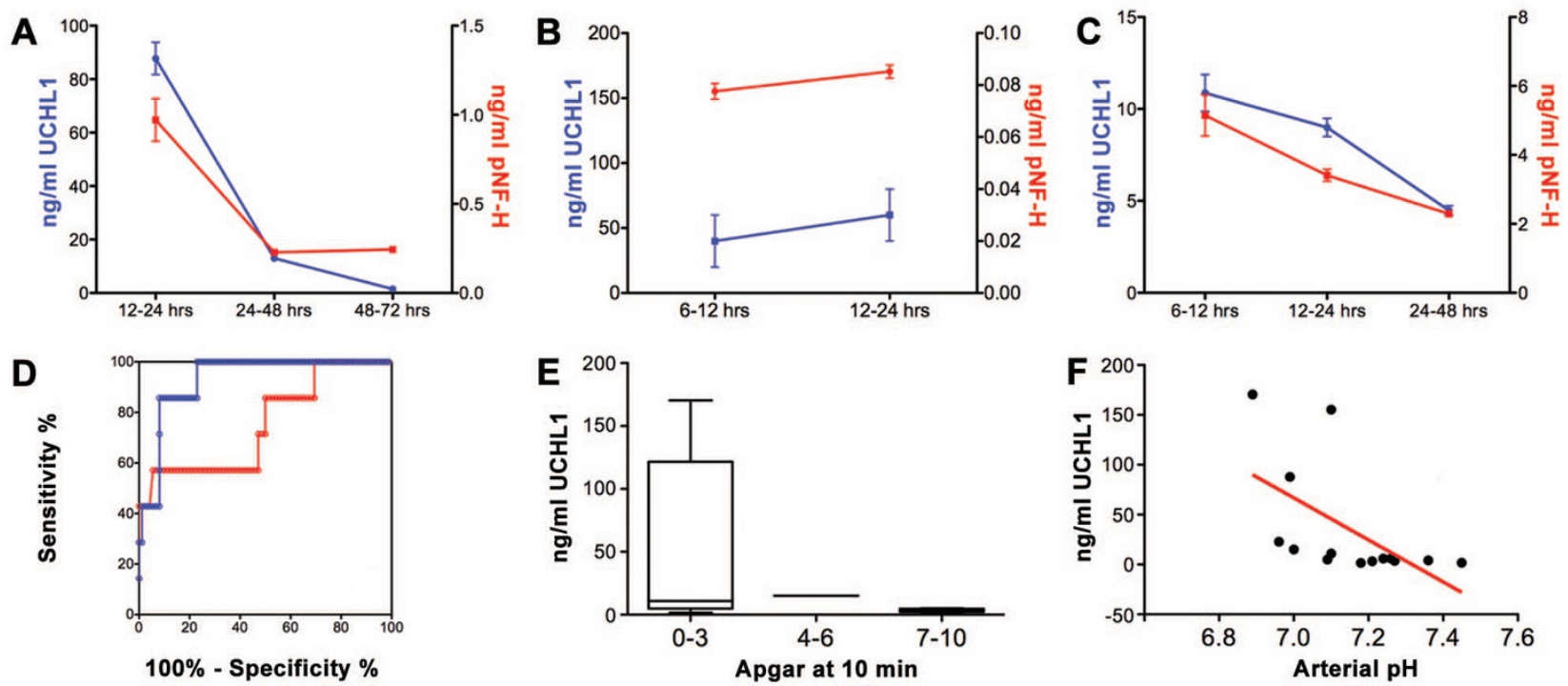

Figure 2. Selected data from individual patients, ROC curves, and relationship between initial serum UCHL1 level, Apgar score, and arterial pH. Panels ( $A-C)$ display data from the three neonatal patients with HIE, who died during this study. Serum levels of UCHL1 are shown in blue, with the scale on the left. Serum levels of pNF-H are shown in blue, with the scale on the right. Error bars are one SD. Panel $(D)$ shows ROC curve for all data collected, with UCHL1 in blue and pNF-H in red. Panel $(E)$ shows box and whisker plots of UCHL1 levels and Apgar score at 10 min, and panel $(F)$ shows relationship between the initial UCHL1 blood levels and arterial $\mathrm{pH}$.

Table 3. Biomarkers correlation with clinical and MRI findings

\begin{tabular}{|c|c|c|c|c|c|c|}
\hline Patient & $\begin{array}{l}\text { pNF-H } \\
\text { (time) }\end{array}$ & $\begin{array}{l}\text { UCHL1 } \\
\text { (time) }\end{array}$ & $\begin{array}{l}\text { 1, 5, and } 10 \mathrm{~min} \\
\text { Apgar }\end{array}$ & Initial $\mathrm{pH}$ & Initial lactate & MRI \\
\hline 1 & $0.63(25-48)$ & $4.85(25-48)$ & $3 / 5 / 8$ & 7.09 & 5.8 & Cortical and thalamus \\
\hline 2 & $0.97(13-24)$ & $87.87(13-24)$ & $1 / 1 / 2$ & 6.99 & 2.2 & $\begin{array}{l}\text { Blood in the ventricles, large extracranial hematomas, } \\
\text { and died }\end{array}$ \\
\hline 3 & $0.43(25-48)$ & $3.73(13-24)$ & $1 / 3 / 3$ & 7.27 & 8.09 & Cortical \\
\hline 4 & $0.45(13-24)$ & $22.71(13-24)$ & $0 / 0 / 1$ & 6.96 & $>20$ & Diffuse, cortical, and subcortical \\
\hline 5 & $0.39(13-24)$ & $5.84(49-72)$ & & 7.24 & 8.4 & Normal \\
\hline 6 & $0.03(7-12)$ & $170.42(7-12)$ & $0 / 0 / 0$ & 6.89 & 13.9 & Died (no MRI) \\
\hline 7 & $0.62(25-48)$ & $3.16(25-48)$ & $2 / 7$ & 7.21 & 13.6 & $\begin{array}{l}\text { Basal ganglia/hippocampus subcortical white matter anc } \\
\text { perirolandic cortex }\end{array}$ \\
\hline 8 & $0.62(13-24)$ & $15.12(13-24)$ & $0 / 2 / 4$ & 7.0 & 19 & Basal ganglia/hippocampus \\
\hline 9 & $0.8(49-72)$ & $155.23(0-6)$ & $0 / 0 / 0$ & 7.1 & 11 & Normal \\
\hline 10 & $0.23(49-72)$ & $6.03(7-12)$ & $2 / 1 / 1$ & 7.26 & 10.8 & Normal \\
\hline 11 & $0.49(48-72)$ & $1.56(7-12)$ & $1 / 1 / 3$ & 7.18 & 10 & Diffuse cortical \\
\hline 12 & $4.6(49-72)$ & $1.81(25-48)$ & $1 / 6 / 7$ & 7.45 & 2.2 & Basal ganglia/hippocampus \\
\hline 13 & $5.15(0-6)$ & $10.88(0-6)$ & $0 / 0 / 0$ & 7.1 & $>20$ & Died \\
\hline 14 & $2.81(49-72)$ & $4.05(49-72)$ & $7 / 9$ & 7.36 & 2.4 & Diffuse cortical \\
\hline
\end{tabular}

The highest level of pNF-H and UCHL1 are shown along with the Apgar scores, initial neonatal arterial pH, lactate, and MRI results. The patients 8, 9, 10, 11 , and 13 received hypothermia. Patients 2, 6, and 13 died. The time of the highest level of each biomarker is shown in parenthesis next to the value.
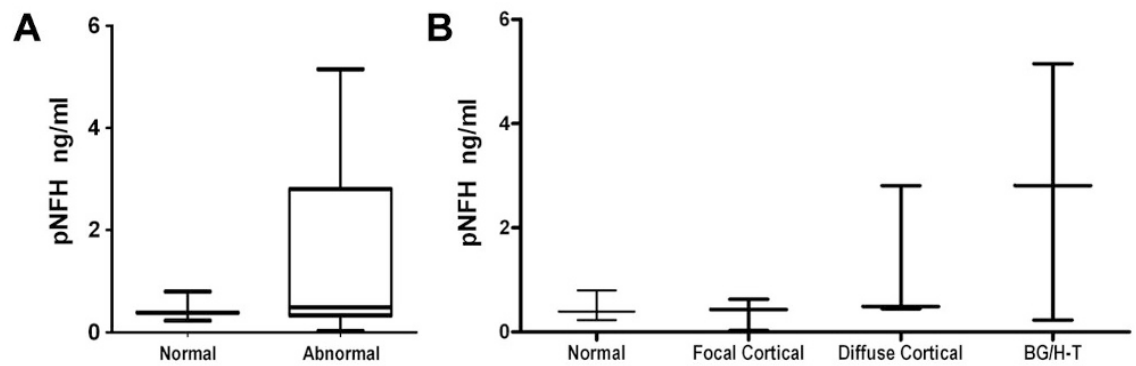

Figure 3. MRI and levels of serum pNF-H. Panel (A) shows pNF-H levels as a function of normal vs abnormal MRI. Panel (B) shows the same data divided into four categories: diffuse cortical, focal cortical, basal ganglia/hippocampus/thalamus (BG/H-T), and normal.
HIE, and assays based on this may have clinical utility in future. UCHL1 levels have also been shown to be increased in the CSF of patients recovering from aneurysmal subarachnoid hemorrhage (12), those with surgically induced circulatory arrest for aortic aneurysm repair (13), and in patients recovering from traumatic brain injury. The detection of large amounts of serum UCHL1 has obvious advantages over CSF UCHL1. Obtaining CSF is technically demanding, can be 
painful, and has a risk of infection, whereas blood samples are part of routine care on very sick neonates.

Interpretation of brain MR scans in neonates with HIE could be challenging and requires an experienced neuroradiologist. The findings can be subtle in the first few days of life, especially when there is diffuse, symmetric brain injury. Heavy reliance must be placed on the diffusion-weighted images, which can be abnormal within hours of injury, as conventional T1- and T2-weighted images are often normal. At several months of age, the atrophy resulting from the brain injury is often readily apparent on MRI.

This work also suggests that pNF-H detection may also have some clinical utility. The ROC analysis shows that, while UCHL1 seems superior, pNF-H also detects brain injury. $\mathrm{NF}-\mathrm{H}$ is a neurofilament protein and is a very attractive biomarker, because it is specific to neural tissues where the heavily phosphorylated form, pNF-H, is axon specific $(6,7)$. The levels of serum pNF-H have been found to be increased in experimental models of spinal cord and traumatic brain injury $(7,11)$ and in patients recovering from aneurysmal subarachnoid hemorrhages or suffering from amyotrophic lateral sclerosis (ALS) or Leber's hereditary optic neuropathy (8-10). The levels of pNF-H within the CNS have been shown to vary according to the brain region with low levels in cerebral cortex and intermediate to high levels found in regions of the brain rich in large diameter axons such as the brain stem, cerebellum, thalamus, subcortical white matter, and basal ganglia $(7,11)$. This may explain why the highest levels of serum pNF-H we observed were in patients with compromise to the basal ganglia, hippocampus, and thalamus detectable by MRI, whereas lower levels were associated with cortical compromise (Fig. 3). The peak level of serum pNF-H in this study $(5 \mathrm{ng} / \mathrm{mL})$ was lower than the peak levels seen in adult acute subarachnoid hemorrhage $(7 \mathrm{ng} / \mathrm{mL})$ and ALS (6 $\mathrm{ng} / \mathrm{mL}$ ) patients $(8,10)$. Because the relative volume of the brain when compared with the body and blood volume is much higher in neonates then in adults, this result may seem surprising. However, NF-H is expressed significantly later in the mammalian brain development than in the other major neurofilament subunits such as NF-L and NF-M (18). Furthermore, the phosphorylated axonal form of NF-H and pNF-H appears later than the nonphosphorylated form and in the rat brain development that is not present at the adult level compared with the other neurofilament subunits until well after birth (19). There is little information on the levels of pNF-H in human brain development, but it is likely that the developing neonatal brain has lower levels of pNF-H than the adult.

Control levels of pNF-H and UCHL1 in normal neonatal serum were $0.21 \pm 0.13 \mathrm{ng} / \mathrm{mL}$ and $2.1 \pm 1.3 \mathrm{ng} / \mathrm{mL}$, respectively. The pNF-H levels are comparable with those seen in control samples seen in two previous human studies. Levels were $0.11 \pm 0.08 \mathrm{ng} / \mathrm{mL}$ in a small group of healthy mostly younger adults (10) and $0.17 \pm 0.09 \mathrm{ng} / \mathrm{mL}$ in 19 individuals who were age-matched controls for ALS patients (8). There are no available reports about serum UCHL1 in healthy controls neonates, although we previously proposed a conservative $95 \%$ cutoff level of $1.89 \mathrm{ng} / \mathrm{mL}$ for adult patients with aneurysmal subarachnoid hemorrhage (12). It is not clear whether the small signal generally seen in control serum samples in this study is because of a small amount of both proteins normally being present in blood or because of the inherent background of the assays. Work in progress is optimizing both assays to address this issue. There is an overlap in blood biomarker level between the control and patient groups with both biomarkers (e.g. Fig. $1 A$ and $B$ ), which may be partly because of this small background. It is also possible that only a fraction of neonatal patients with HIE release UCHL1 and pNF-H into the blood in obviously increased amounts. Only further studies will resolve these issues.

We recognize that an effective biomarker should have a high correlation with long-term outcomes (1). Few serum markers, such as IL-6, IL-8, and ionized calcium, have shown promise in predicting long-term outcomes after HIE (1). Other potential biomarkers are $\mathrm{S}-100 \beta$ in urine and neuron-specific enolase and glial fibrillary acidic protein in the CSF (1). Given the lack of a clinically usable serum biomarker and the limitations of this study, we were encouraged by our results. Both UCHL1 and pNF-H are expressed only in neurons and so may reflect brain injury in a much more specific fashion than IL6, IL-8, and calcium that may be released after a variety of inflammatory challenges. Because both UCHL1 and pNF-H were detected at the earliest time point, $0-6 \mathrm{~h}$, they are potentially useful in detecting the degree of injury before initiating neuroprotective strategies such as hypothermia. The large amount of UCHL1 detected in some samples coupled with the stability and abundance of this protein suggests that rapid assays, perhaps filter based, should be straightforward to develop. Furthermore, the ratio of UCHL1 and pNF-H may reveal the relative loss of cortical versus white matter tissue. Larger studies are clearly needed to examine the ability of the 0 to $6 \mathrm{~h}$ serum levels of pNF-H and UCHL1 to identify neonates with injury when compared with controls, to predict the neuroimaging pattern found on MRI, and to predict longterm outcomes. As neuroprotective strategies in neonates with HIE evolve, the stratification and initial objective assessment of these patients should also evolve. Serum biomarkers offer the hope of providing objective information about the initial degree of injury with the added benefit of potentially providing a surrogate marker of treatment efficacy.

This study has several limitations. First, it was a pilot study with a small patient sample size. Second, our study was designed to look at the neuroimaging findings as a short-term outcome measure. This was done in an attempt to develop biomarkers that would predict the brain pattern of injury at a very early time point before potential neuroprotective strategies are begun. Because of the small sample size, we were only able to perform a descriptive analysis for the imaging results. Third, we do not have data on long-term outcomes. We hope to address all these issues in future in more extensive studies on larger numbers of neonatal patients with HIE. In conclusion, our findings suggest that serum UCHL1 and pNF-H should be further explored as diagnostic and prognostic tools in neonatal HIE. 


\section{REFERENCES}

1. Ramaswamy V, Horton J, Vandermeer B, Buscemi N, Miller S, Yager J 2009 Systematic review of biomarkers of brain injury in term neonatal encephalopathy. Pediatr Neurol 40:215-226

2. Thorngren-Jerneck K, Alling C, Herbst A, Amer-Wahlin I, Marsal K 2004 S100 protein in serum as a prognostic marker for cerebral injury in term newborn infants with hypoxic ischemic encephalopathy. Pediatr Res 55:406-412

3. Sarnat HB, Sarnat MS 1976 Neonatal encephalopathy following fetal distress. A clinical and electroencephalographic study. Arch Neurol 33:696-705

4. Gunn AJ, Gunn TR, Gunning MI, Williams CE, Gluckman PD 1998 Neuroprotection with prolonged head cooling started before postischemic seizures in fetal sheep. Pediatrics 102:1098-1106

5. Gunn AJ 2000 Cerebral hypothermia for prevention of brain injury following perinatal asphyxia. Curr Opin Pediatr 12:111-115

6. Shaw G 1998 Neurofilaments. Springer, New York, NY

7. Anderson KJ, Scheff SW, Miller KM, Roberts KN, Gilmer LK, Yang C, Shaw G 2008 The phosphorylated axonal form of the neurofilament subunit NF-H (pNF-H) as a blood biomarker of traumatic brain injury. J Neurotrauma 25:1079-1085

8. Boylan K, Yang C, Crook J, Overstreet K, Heckman M, Wang Y, Borchelt D, Shaw G 2009 Immunoreactivity of the phosphorylated axonal neurofilament $\mathrm{H}$ subunit (pNF-H) in blood of ALS model rodents and ALS patients: evaluation of blood pNF-H as a potential ALS biomarker. J Neurochem 111:1182-1191

9. Guy J, Shaw G, Ross-Cisneros FN, Quiros P, Salomao SR, Berezovsky A, Carelli V, Feuer WJ, Sadun AA 2008 Phosphorylated neurofilament heavy chain is a marker of neurodegeneration in Leber hereditary optic neuropathy (LHON). Mol Vis 14:24432450
10. Lewis SB, Wolper RA, Miralia L, Yang C, Shaw G 2008 Detection of phosphorylated NF-H in the cerebrospinal fluid and blood of aneurysmal subarachnoid hemorrhage patients. J Cereb Blood Flow Metab 28:1261-1271

11. Shaw G, Yang C, Ellis R, Anderson K, Parker Mickle J, Scheff S, Pike B, Anderson DK, Howland DR 2005 Hyperphosphorylated neurofilament NF-H is a serum biomarker of axonal injury. Biochem Biophys Res Commun 336:1268-1277

12. Lewis SB, Wolper R, Chi YY, Miralia L, Wang Y, Yang C, Shaw G 2010 Identification and preliminary characterization of ubiquitin $\mathrm{C}$ terminal hydrolase 1 (UCHL1) as a biomarker of neuronal loss in aneurysmal subarachnoid hemorrhage. J Neurosci Res 88:1475-1484

13. Siman R, Roberts VL, McNeil E, Dang A, Bavaria JE, Ramchandren S, McGarvey M 2008 Biomarker evidence for mild central nervous system injury after surgicallyinduced circulation arrest. Brain Res 1213:1-11

14. Siman R, Toraskar N, Dang A, McNeil E, McGarvey M, Plaum J, Maloney E, Grady MS 2009 A panel of neuron-enriched proteins as markers for traumatic brain injury in humans. J Neurotrauma 26:1867-1877

15. Thompson RJ, Doran JF, Jackson P, Dhillon AP, Rode J 1983 PGP 9.5-a new marker for vertebrate neurons and neuroendocrine cells. Brain Res 278:224-228

16. Wilkinson KD, Lee KM, Deshpande S, Duerksen-Hughes P, Boss JM, Pohl J 1989 The neuron-specific protein PGP 9.5 is a ubiquitin carboxyl-terminal hydrolase. Science 246:670-673

17. Harris J, Ayyub C, Shaw G 1991 A molecular dissection of the carboxyterminal tails of the major neurofilament subunits NF-M and NF-H. J Neurosci Res 30:47-62

18. Shaw G, Weber K 1982 Differential expression of neurofilament triplet proteins in brain development. Nature 298:277-279

19. Carden MJ, Trojanowski JQ, Schlaepfer WW, Lee VM 1987 Two-stage expression of neurofilament polypeptides during rat neurogenesis with early establishment of adult phosphorylation patterns. J Neurosci 7:3489-3504
United States Postal Service

Statement of Ownership, Management, and Circulation

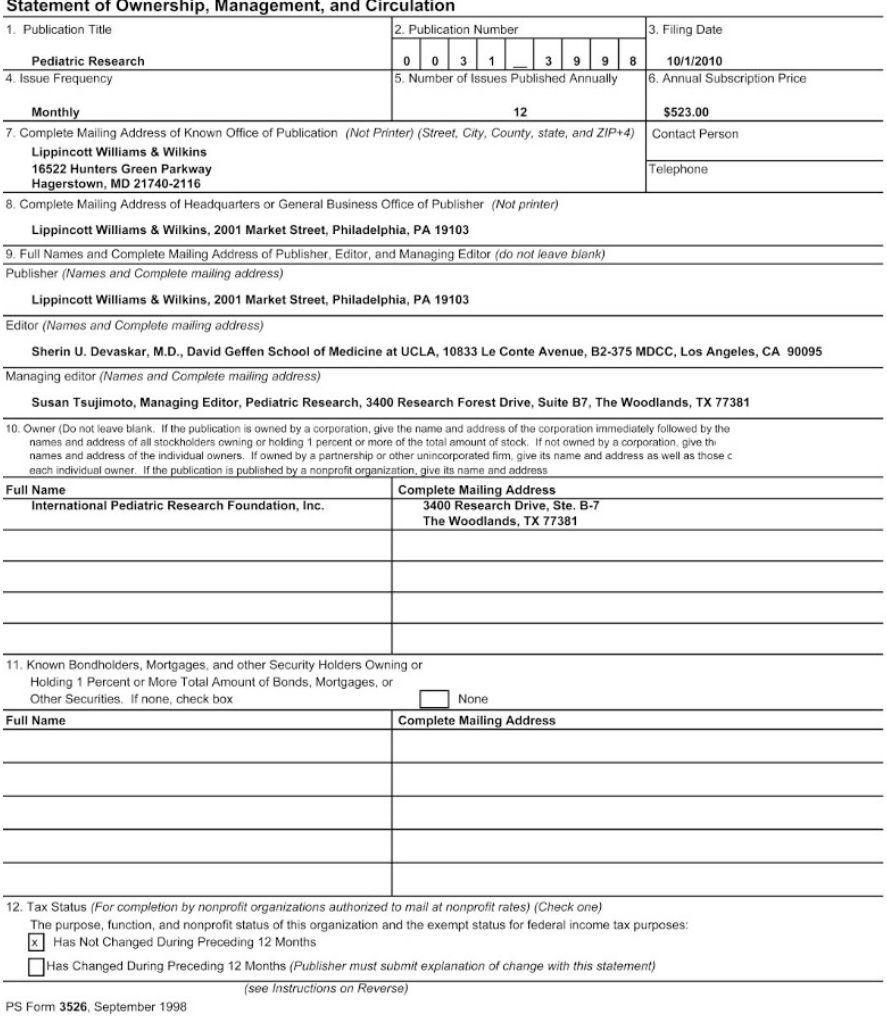

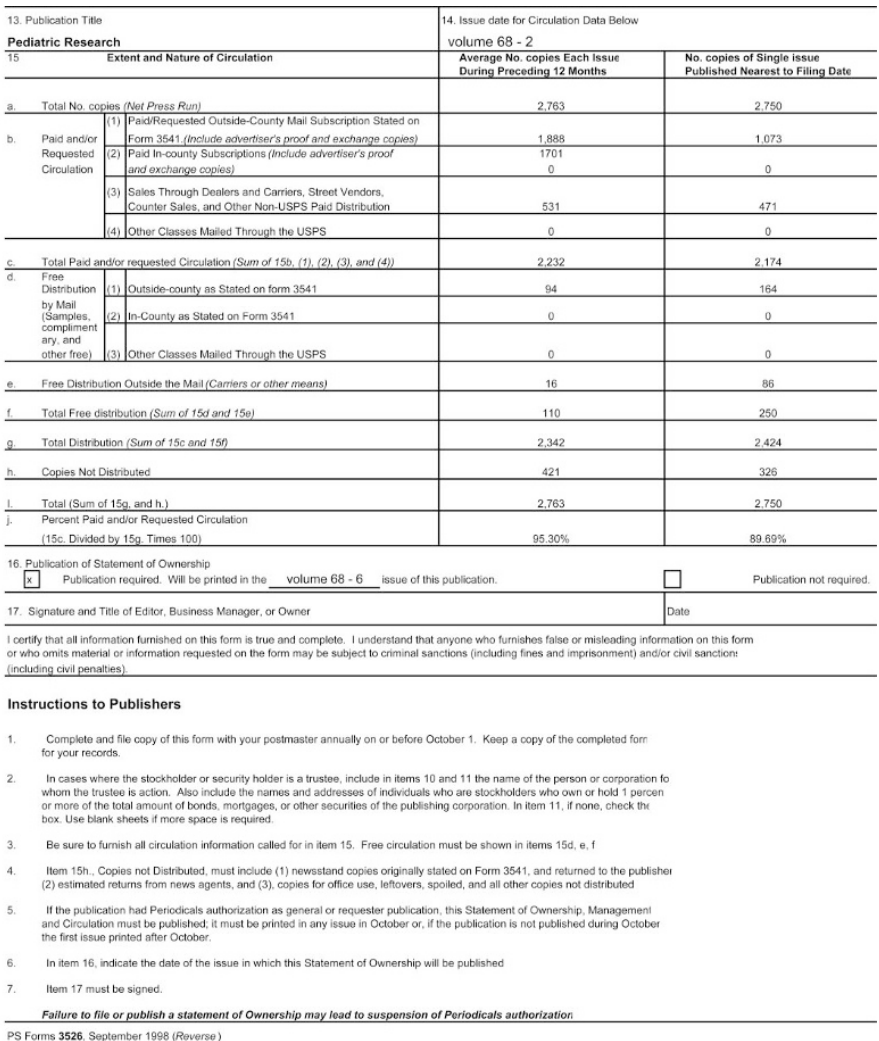

